\title{
Towards a full and realistic simulation framework for the Extreme Energy Events experiment
}

\section{S. Grazzi on behalf of the EEE Collaboration ${ }^{a, b, \dagger, *}$}

${ }^{a}$ Dipartimento di Scienze Matematiche e Informatiche, Scienze Fisiche e Scienze della Terra, Università di Messina, Messina, Italy

${ }^{b}$ INFN Sezione di Genova, Genova, Italy

E-mail: stegrazzi@unime.it, Stefano.Grazzi@ge.infn.it

The network of MRPC (Multi-gap Resistive Plate Chambers) telescopes of the Extreme Energy Events experiment (EEE) was designed to study very high energy cosmic rays through detection of secondary cosmic muons in the hadronic shower. To better understand and predict the behavior of such events, a GEANT4-based simulation framework that well reproduces the response of individual telescopes was built. Simulations are crucial to better understand the detectors performance in current setup and how these are affected by the specific installations. This is the first step towards a full simulation framework that includes a realistic generation, now limited to muons, of secondary particles and propagation through the atmosphere of the shower produced by primary rays. The current framework can be used to characterize and optimize the array of EEE telescopes, simulating not only the single detectors but also telescope's clusters, providing insight in extreme energy and rare events. In this contribution, the EEE simulation framework and future plans will be presented.

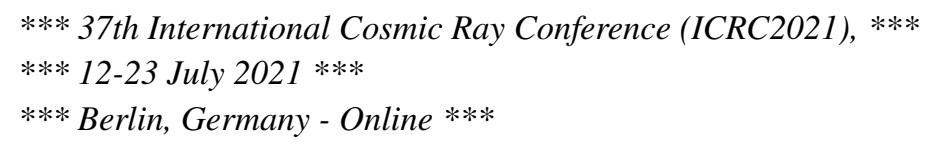

\footnotetext{
*Speaker

$\dagger$ Complete list of authors can be found at the end of the proceedings
} 


\section{Introduction}

The Extreme Energy Events (EEE) experiment $[1,2]$ deployed a network with about 60 cosmic muon detectors dislocated in an area of $3 \times 10^{5} \mathrm{~km}^{2}$ over Italian Territory. The EEE network precisely measures cosmic muon rates and arrival times for search very rare events generated in far universe. Some recent results, published by the EEE Collaboration, include: observation of the Forbush effect [3], searches for anisotropies in the cosmic ray intensity [4], and long distance correlation in secondary muons [2]. A quantitative understanding of these phenomena can be obtained by a precise measurement of the cosmic muon rates and arrival times. To do so a sophisticated detector, a good understanding of the experimental conditions and an excellent synchronization of data coming from different telescopes in the EEE grid is mandatory. All EEE detectors are based on the MRPC technology similar to ALICE Time-Of-Flight detectors, but with slightly different experimental configurations (e.g. the distance between the chambers and the absolute orientation w.r.t. the North are not always the same). Moreover, the measured rate is affected by the material surrounding the detector that is different for each telescope since they are hosted in rooms located in non-dedicated buildings (high schools or university labs). For a thorough interpretation of experimental observations (cosmic ray absolute rates and angular distributions), a Monte Carlo simulation of the detector's response and experimental conditions is therefore necessary.

\section{The EEE Telescope}

More details of EEE telescope can be found in Ref. [1]. Each station of the EEE network, that defines a "telescope" for cosmic rays (mainly muons), is made of three Multigap Resistive Plate Chambers (MRPC) specifically designed to achieve good tracking and timing capability, low construction costs, and an easy assembly procedure [5]. Three MRPC chambers are placed one above the other with a distance between each chamber, for standard configuration, of $50 \mathrm{~cm}$. Each MRPC consists of 6 gas gaps obtained by stacking glass sheets separated from each other by fishing line spacers, placed inside a gas-tight aluminum box and flushed with a mixture made of $\mathrm{C}_{2} \mathrm{H}_{2} \mathrm{~F}_{4}$ and $\mathrm{SF}_{4}$ in $98 / 2$ proportions. The readout panel is split in 24 copper strips, $180 \mathrm{~cm}$ long and $2.5 \mathrm{~cm}$ wide each, separated one from another by $7 \mathrm{~mm}$ of space, each connected, at the two ends, to a readout channel of the EEE Front-End Amplifier Boards (FEA). The active area is defined by the $158 \mathrm{~cm} \times 82 \mathrm{~cm}$ glass surface. High voltage electrodes placed on the opposite sides of the stack provide an electric field in the gas, strong enough that the ion electron pairs produced by ionizing particles crossing the MRPCs give origin to Townsend avalanches which induce detectable signals on the readout strips.

\subsection{Detector working principle}

A muon track candidate is identified by requiring a valid signal from at least one of the 24 strips connected on each of the six FEA (one for each short sides of the three chambers of a telescope). When this condition is achieved a trigger signal is generated by the EEE Trigger Board [6], and used as a start to the data acquisition. The track's impact point (hit) X-coordinate is derived by measuring the signal arrival time difference at the ends of the readout strip. The hit $\mathrm{Y}$ coordinate is 
obtained by the corresponding strip position or from the middle position in case of crossing between two adjacent strips. To improve synchronization between MRPCs, a common clock is generated by a custom VME. Synchronization between telescopes, sometime at distances of a few hundred meters in the same town, sometimes located hundreds of km away, is ensured by a GPS unit. The EEE MRPC telescopes show good performance in terms of efficiency of tracks reconstruction, time resolution $\left(\sigma_{T} \approx 238 \mathrm{ps}\right)$, and spatial resolution $\left(\sigma_{S_{L}} \approx 1.5 \mathrm{~cm}\right.$ and $\sigma_{S_{T}} \approx 0.92 \mathrm{~cm}$ for longitudinal and transverse directions, respectively) [7].

\section{Simulation Framework}

A detailed description of the MRPC geometry and materials has been implemented in GEMC [9], a GEANT4 libraries-based interface with user-defined geometry and hits description [8]. The three chambers forming a telescope are positioned in a stack with a variable distance reproducing the experimental setup of a specific telescope. To mimic a generic room where a real telescope is located, the three chambers are inserted in a box whose walls are made of concrete with variable thickness. The nominal configuration corresponds to a thickness of $30 \mathrm{~cm}$ for each of the six box walls. This is the simplest configuration for an EEE telescope: more complicated geometries of the surrounding materials have also been implemented, for instance, by considering the foundations of multi-floors buildings and/or nearby obstacles, such as other buildings or natural structures. More detail about simulation framework can be found in Ref.[10].

\subsection{Detector response}

The MRPC response was parametrized based upon the measured performance of the chambers. The ionization avalanche in the gas produced by a crossing charged particle was effectively described by considering its effect on the sensitive area of the detector, namely the readout plane, segmented in $2.5 \mathrm{~cm}$ copper strips. The strip multiplicity, defined as the number of contiguous firing strips per particle crossing, was obtained by centering an ellipse in the intersection point of the track with the strip plane. The ellipse sizes were assumed to be the measured cluster sizes $\sigma_{S_{L}}$ and $\sigma_{S_{T}}$ [7]. In case the ellipse covers two or more adjacent strips, all of them are assumed to fire. This procedure takes into account multiple-hits per event recorded by the telescope. The hit time was propagated to the strip sides by considering the measured signal propagation velocity along the strip $(15.8 \mathrm{~cm} / \mathrm{ns})$ with a Gaussian resolution of $\sigma_{T}=238$ ps.

\subsection{Cosmic muon flux parametrization}

Single-muon events are generated according to the model described in Ref.[11]. While the azimuthal $\phi_{\mu}$ distribution is considered to be uniform, the polar angle distribution ( $\theta_{\mu}$ being the angle with respect to the vertical) is reproduced using an improved [12] Gaisser-like parametrization [13] that includes the Earth curvature at all latitudes and low energy muons $\left(E_{\mu}<100 \mathrm{GeV}\right)$. As shown in Ref. [12], this parametrization reproduces quite well the existing measurements. To take into account any possible material surrounding the telescope implemented in the GEANT model (concrete wall, roof, nearby buildings), once the track is generated, the vertex is moved far upwards, ( $\sim 80 \mathrm{~m}$ away), well outside any volumes present in the simulation. Generated muons were then 
passed to GEMC to obtain the EEE telescope expected angular distribution and counting rate of single-track events.

\section{Simulation framework validation}

The EEE simulation framework has been validated by comparing MC predictions with data measured by EEE telescopes. The TORI-03 experimental setup was implemented in GEMC
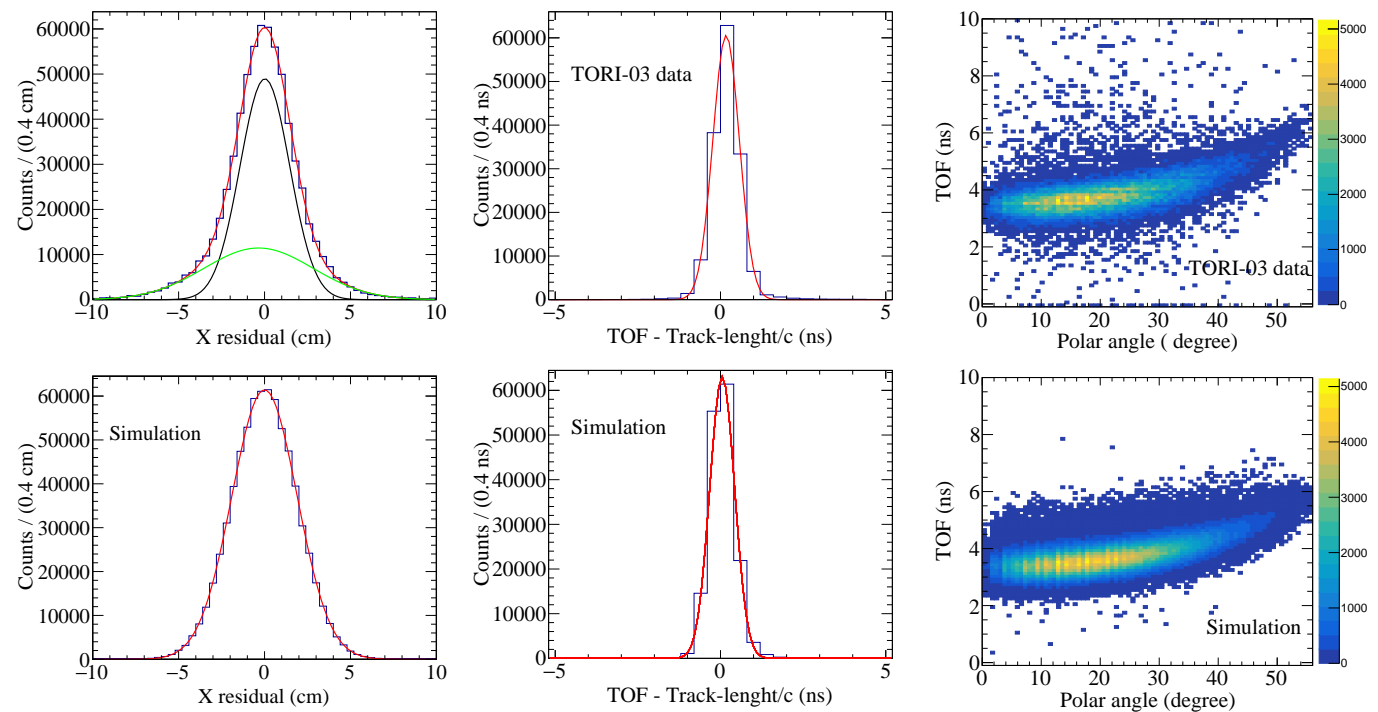

Figure 1: Top Figures: TORI-03 telescope track X coordinate residual (left), Time-of-Flight - track length over $c$ (with respect to the speed of light) (middle), correlation between tracks Time-of-Flight and polar angle (right). In the top (middle) panel, the standard deviation of the Gaussian fits are: $\sigma_{\mathrm{X}}=(1.4 \pm 0.3) \mathrm{cm}$, $\sigma_{\mathrm{TOF}-\mathrm{TrL}}=(0.37 \pm 0.03) \mathrm{ns}$, respectively. Bottom Figures:TORI-03 telescope simulation. Observables are the same as Top. The standard deviation of the Gaussian fits are: $\sigma_{\mathrm{X}}=(1.90 \pm 0.15) \mathrm{cm}, \sigma_{\mathrm{TOF}-\mathrm{TrL}}=$ $(0.40 \pm 0.05) \mathrm{ns}$, respectively.

using the standard geometry and surrounding materials. Experimental data have been corrected by weighting each event with an efficiency map derived by data, to take into account effects not included in the simulations. Both the parametrization of detector response and the model used to generate cosmic muons contribute to the reported observables. Figure 1 shows the comparison of some observables derived from experimental data and simulations. The ratio between distribution of single-muon rates measured by TORI-03 and the ones predicted by simulations, as a function of the muon track polar angle, is shown in Fig 2. The good agreement at all angles and the mean value of the ratio, resulting to be around unity, demonstrate that the EEE simulation framework is able to reproduce the absolute observed angular cosmic muon rate within an error of about $5 \%$ for polar angle lower than $25^{\circ}$ and of about $10 \%$ for polar angle lower than $38^{\circ}$. Similar agreement was obtained for other EEE telescope. This demonstrates that the EEE simulation framework reproduces well the experimental data and can be used to compare telescopes featuring different setups. 


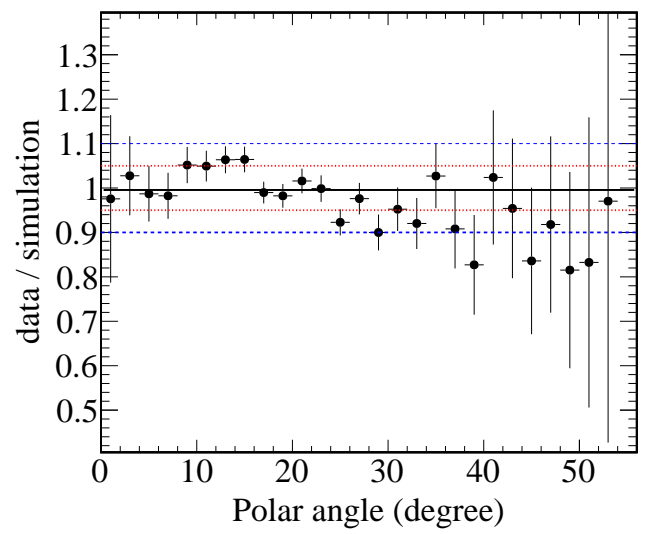

Figure 2: Comparison between cosmic muon rate as a function of the track polar angle for TORI-03 data (red open circles) and the ones predicted by simulations (blue full circles). The ratio between the two distributions is shown in the bottom panel. The red (blue) line delimits a $\pm 5 \%( \pm 10 \%)$ band, the solid (black) line corresponds to a fit with a constant function found to be $1.00 \pm 0.01$.

\section{Systematic studies}

The simulation framework has been used to assess EEE telescope's performance studying: angular and spatial resolution, detection efficiency, and the effects of surrounding materials.

\subsection{Resolution}

The telescope polar angular resolution has been evaluated by generating muons according to the improved Gaisser energy distribution reported in Eq. (1), in the (10-100) GeV energy range to minimize multiple scattering of crossing particles. The telescope was simulated with standard MRPC distance $(50 / 50 \mathrm{~cm})$. The angular resolution $\sigma$ as a function of the track polar angle $\theta$

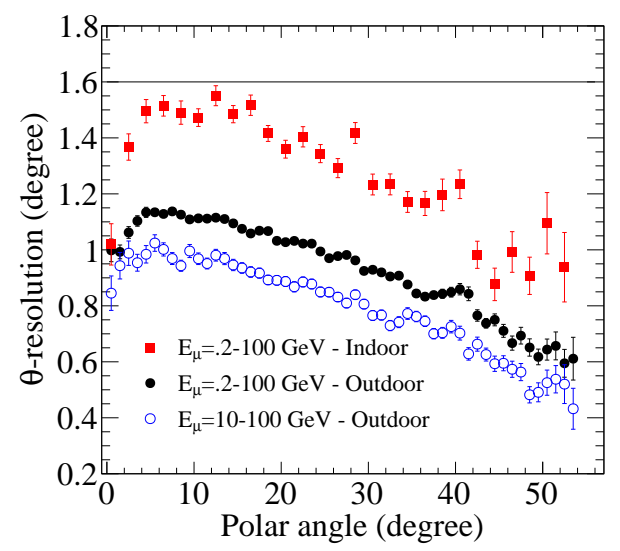

Figure 3: EEE telescope angular resolution as a function of the track polar angle for different muon energy ranges and location conditions.

is shown in Fig. 3. As expected, due to multiple scattering, low-energy muons worsen angular resolution up to 50\%. Data show the best resolution is found at the edges of the angular acceptance. This is a non-trivial result, within the EEE simulations framework. Other results can be found in Ref. [10]. 


\subsection{Efficiency}

The relative detection efficiency, obtained as the ratio of detected and generated muons, as a function of the polar angle for an outdoor standard configuration $(50 / 50 \mathrm{~cm})$ telescope, and muons generated in the full energy range $(0.2-100 \mathrm{GeV})$ is shown in left pannel of Fig. 4. The distribution has been normalized to the maximum value corresponding to vertical tracks. The relative detection efficiency monotonically decreases as the track polar angle increases. The relative
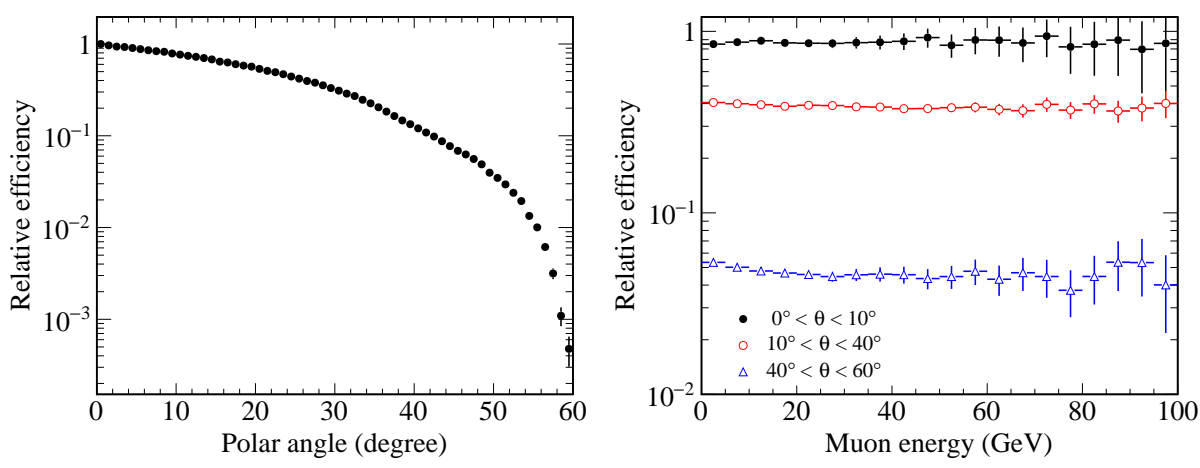

Figure 4: On left: Detection efficiency as a function of the muon track polar angle for a standard configuration of an outdoor telescope. On Right: Detection efficiency as a function of muon energy for different intervals of the track polar angle for a standard configuration of an outdoor telescope.

detection efficiency as a function of muon energy for different intervals of track polar angle is shown in right panel of Fig. 4. The distributions are normalized to the maximum value corresponding to $\theta=0^{\circ}$. The relative detection efficiency shows a weak dependence on the muon energy for all angular intervals.

\subsection{Effects of surrounding materials}

EEE telescopes are often placed in rooms with variable thickness of concrete walls and roof. For a correct comparison of results obtained by different telescopes in the EEE network, it is important to evaluate the effect of the materials surrounding the telescope. Simulations provide a simple end effective tool to understand and correct for these effects. We studied different experimental setups, common to the EEE network, with an increasing complexity of the environmental conditions, including the effect of a realist model for windows.

Some,results are shown in Fig. 5. From these studies it can be seen that even small details can significantly influence angular the distributions. These variations are not always trivial but must be taken into account to minimize the systematic error data analysis. Such a significant sensitivity is an interesting feature that demonstrates how muons can be used to perform a scan of surrounding materials (muon tomography) [14]. Further investigations are in progress. A more complex case concerns the telescope installed in the Physics Department of the University of Genova (GENO-01). This is a particular tricky geometry, since the telescope is located under many floors and the building is surrounded by a mountain on one side and a valley on the other, defining an asymmetric shielding for cosmic rays.

Figure 6 shows the experimental asymmetry as measured by the GENO-01 telescope. The asymmetric absorption of muons due to the building structure may explain the observed asymmetry. 

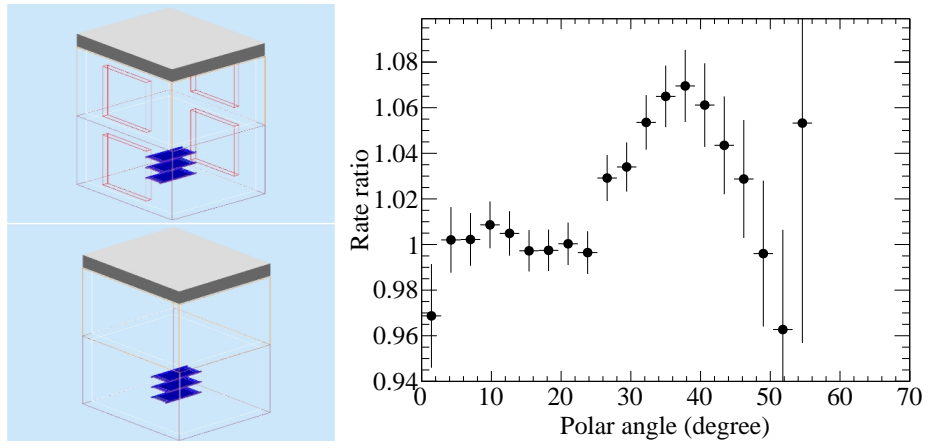

Figure 5: On right: ratio between the two rate distributions for an indoor telescope hosted in a building with large windows and without. On left: the two simulated geometries.
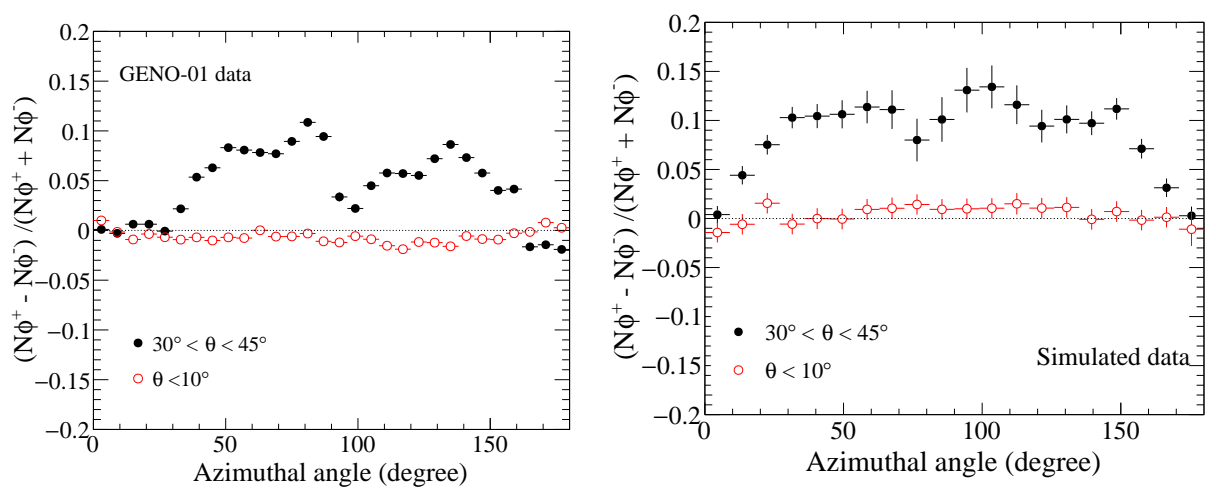

Figure 6: On Left: GENO-01 telescope experimental asymmetry for muon tracks with $30^{\circ}<\theta<45^{\circ}$ (full circles), with $\theta<10^{\circ}$ (open circles). On Right: the results of the same $\phi$ asymmetry obtained with simulation.

This configuration was implemented in the simulation framework by placing the detector below a $70 \mathrm{~cm}$-thick concrete asymmetric roof (mimicking the asymmetric structure of the building) and placing on one side a volume that absorbs muons (mimicking the side hill). The GEMC implementation and the resulting asymmetry are shown in Fig 6. Simulations are able to reproduce the shape and the magnitude of the experimental asymmetries for $\theta<10^{\circ}$ and $30^{\circ}<\theta<45^{\circ}$.

\section{Conclusions}

The EEE simulation framework is a valuable tool to study the detector performance, it can be used to compare and correct the response of different detectors in the network in order to achieve the systematic precision necessary to study of small effects such as the variations of the cosmic ray flux. In future we plan to integrate in the framework CORSIKA event generator for a detailed simulation of extensive air showers initiated by high energy primaries. CORSIKA and the inclusion of cosmic ray cluster tracking will provide a more realistic framework to understand the angular and energy distributions and of the events detected by the network of telescopes. Finally, we showed that EEE simulation framework can be used to investigate new opportunities, such as the use of cosmic muons for building tomography, extending the current scope of the EEE Collaboration. 


\section{References}

[1] M. Abbrescia et al. (EEE Collaboration), The EEE experiment project: status and first physics results, Eur. Phys. J. Plus 128, 86 (2013).

[2] M. Abbrescia et al. (EEE Collaboration), Search for long distance correlations between extensive air showers detected by the EEE network, Eur. Phys. J. Plus 133, 34 (2018).

[3] M. Abbrescia et al. (EEE Collaboration), Observation of the February 2011 Forbush decrease by the EEE telescopes, Eur. Phys. J. Plus (2011) 126, 61.

[4] M. Abbrescia et al. (EEE Collaboration), Looking at the sub-TeV sky with cosmic muons detected in the EEE MRPC telescopes, Eur. Phys. J. Plus 130 (2015) 187.

[5] M. Abbrescia et al., Performance of a six gap MRPC built for large area coverage, Nucl. Instrum. Meth. A 593, 263 (2008).

[6] Panetta M.P. et. al. (EEE Collaboration), The new trigger/GPS module for the EEE project, Nucl. Instrum. Meth. A 936, 376 (2018).

[7] EEE Collaboration, The Extreme Energy Events experiment: an overview of the telescopes performance, Published by IOP Publishing for Sissa Medialab, JINST 13 (2018) 08, P08026.

[8] GEANT4, a simulation toolkit: https://geant4.web.cern.ch/

[9] GEMC, GEant4 Monte-Carlo (version 2.6): https://gemc.jlab.org/gemc/html/index.html/

[10] M. Abbrescia et al. The cosmic muon and detector simulation framework of the extreme energy events (eee) experiment. 2021. arXiv:2104.06012.

[11] H. M. Kluck, Measurement of the Cosmic-Induced Neutron Yield at the Modane Underground Laboratory, Ph.D. thesis, KIT, Karlsruhe (2013). doi:10.1007/978-3-319-18527-9.

[12] M. Guan, M. C. Chu, J. Cao, K. B. Luk, C. Yang, A parametrization of the cosmic ray muon flux at sea-level, arXiv e-prints (2015) arXiv:1509.06176.

[13] T. Gaisser, T. Stanev, Cosmic Rays in Review of Particle Physics, Physics Letters B 592 (2018). URL http://pdg.lbl.gov

[14] F. Riggi et al., Multiparametric approach to the assessment of muon tomographic results for the inspection of a full-scale container, Eur. Phys. J. Plus 136, 139 (2021). 


\section{Full Authors List: EEE Collaboration}

M. Abbrescia ${ }^{1,2}$, C. Avanzini ${ }^{3}$, L. Baldini ${ }^{3,4}$, R. Baldini Ferroli ${ }^{5}$, G. Batignani ${ }^{3,4}$, M. Battaglieri ${ }^{6,7}$, S. Boi ${ }^{8,9}$, E. Bossini ${ }^{3,4}$, F. Carnesecchi ${ }^{10,11}$, C. Cicalò ${ }^{9}$, L. Cifarelli ${ }^{10,11}$, F. Coccetti ${ }^{12}$, E. Coccia ${ }^{13}$, A. Corvaglia ${ }^{14}$, D. De Gruttola ${ }^{15,16}$, S. De Pasquale ${ }^{15,16}$, F. Fabbri ${ }^{5}$, L. Galante ${ }^{17,18}$, M. Garbini ${ }^{10,12}$, G. Gemme ${ }^{6}$, I. Gnesi ${ }^{12,19}$, S. Grazzi ${ }^{6,20}$, D. Hatzifotiadou ${ }^{10,21}$, P. La Rocca ${ }^{22,23}$, Z. $\mathrm{Liu}^{24}$, G. Mandaglio ${ }^{20,23}$, G. Maron ${ }^{25}$, M. N. Mazziotta ${ }^{2}$, A. Mulliri ${ }^{8,9}$, R. Nania ${ }^{10}$, F. Noferini ${ }^{10}$, F. Nozzoli ${ }^{26}$, F. Palmonari ${ }^{10,11}$, M. Panareo ${ }^{14,27}$, M. P. Panetta ${ }^{12,14}$, R. Paoletti ${ }^{3,28}$, C. Pellegrino ${ }^{25}$, O. Pinazza ${ }^{10}$, C. Pinto ${ }^{22,23}$, S. Pisano ${ }^{5,12}$, F. Riggi ${ }^{22,23}$, G. C. Righini ${ }^{29}$, C. Ripoli ${ }^{15,16}$, M. Rizzi ${ }^{2}$, G. Sartorelli ${ }^{10,11}$, E. Scapparone ${ }^{10}$, M. Schioppa ${ }^{19,30}$, A. Scribano ${ }^{28}$, M. Selvi ${ }^{10}$, G. Serri ${ }^{8,9}$,

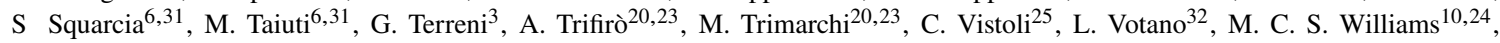
A. Zichichi ${ }^{10,11,21}$ and R. Zuyeuski ${ }^{24}$

${ }^{1}$ Dipartimento Interateneo di Fisica, Università di Bari, Bari, Italy. ${ }^{2}$ INFN Sezione di Bari, Bari, Italy. ${ }^{3}$ INFN Sezione di Pisa, Pisa, Italy. ${ }^{4}$ Dipartimento di Fisica, Università di Pisa, Pisa, Italy. ${ }^{5}$ INFN Laboratori Nazionali di Frascati, Frascati (Rome), Italy. ${ }^{6}$ INFN Sezione di Genova, Genova, Italy. ${ }^{7}$ Thomas Jefferson National Accelerator Facility, Newport News, VA, USA. ${ }^{8}$ Dipartimento di Fisica, Università di Cagliari, Cagliari, Italy. ${ }^{9}$ INFN Sezione di Cagliari, Cagliari, Italy. ${ }^{10}$ INFN Sezione di Bologna, Bologna, Italy. ${ }^{11}$ Dipartimento di Fisica ed Astronomia, Università di Bologna, Bologna, Italy. ${ }^{12}$ Museo Storico della Fisica e Centro Studi e Ricerche "E. Fermi", Rome, Italy. ${ }^{13}$ Gran Sasso Science Institute, LAquila, Italy. ${ }^{14}$ INFN Sezione di Lecce, Lecce, Italy. ${ }^{15}$ Dipartimento di Fisica, Università di Salerno, Salerno, Italy. ${ }^{16}$ INFN Gruppo Collegato di Salerno, Salerno, Italy. ${ }^{17}$ Dipartimento di Scienze Applicate e Tecnologia, Politecnico di Torino, Torino, Italy. ${ }^{18}$ INFN Sezione di Torino, Torino, Italy. ${ }^{19}$ INFN Gruppo Collegato di Cosenza, Cosenza, Italy. ${ }^{20}$ Dipartimento di Scienze Matematiche e Informatiche, Scienze Fisiche e Scienze della Terra, Università di Messina, Messina, Italy. ${ }^{21} \mathrm{CERN}$, Geneva, Switzerland. ${ }^{22}$ Dipartimento di Fisica e Astronomia, Università di Catania, Catania, Italy. ${ }^{23}$ INFN Sezione di Catania, Catania, Italy. ${ }^{24}$ ICSC World laboratory, Geneva, Switzerland. ${ }^{25}$ INFN-CNAF, Bologna, Italy. ${ }^{26}$ INFN Trento Institute for Fundamental Physics and Applications, Trento, Italy. ${ }^{27}$ Dipartimento di Matematica e Fisica, Università del Salento, Lecce, Italy. ${ }^{28}$ Dipartimento di Scienze Fisiche, della Terra e dell'Ambiente, Università di Siena, Siena, Italy. ${ }^{29}$ CNR Istituto di Fisica Applicata "Nello Carrara", Sesto Fiorentino (Florence), Italy. ${ }^{30}$ Dipartimento di Fisica, Università della Calabria, Rende (Cosenza), Italy. ${ }^{31}$ Dipartimento di Fisica, Università di Genova, Genova, Italy. ${ }^{32}$ INFN, Laboratori Nazionali del Gran Sasso, Assergi (L'Aquila), Italy. 